\title{
Stiffness of cargo-motor linkage tunes Myosin VI motility and response to load
}

\author{
Rachit Shrivastava, ${ }^{\dagger, \S}$ Ashim Rai, ${ }^{\ddagger}, \S$ Murti Salapaka, ${ }^{*, \dagger}, \boldsymbol{\Phi}$ and Sivaraj \\ Sivaramakrishnan*,+, \\ $\dagger$ Electrical and Computer Engineering, University of Minnesota, Twin Cities \\ $\ddagger$ Genetics Cell Development and Biology, University of Minnesota, Twin Cities \\ \Authors to whom the correspondences should be addressed \\ $\S$ Authors have contributed equally \\ E-mail: murti@umn.edu; sivaraj@umn.edu
}

\section{Materials and Methods}

\section{Buffer and reagents}

Assay Buffer (AB): $25 \mathrm{mM}$ imidazole (pH 7.5), $4 \mathrm{mM} \mathrm{MgCl} 2,1 \mathrm{mM}$ EGTA, $25 \mathrm{mM} \mathrm{KCl}$, $1 \mathrm{mM}$ DTT; AB.BSA: AB buffer $+1 \mathrm{mg} / \mathrm{ml}$ BSA; AB.BSA.Cam: AB.BSA buffer $+9 \mu \mathrm{M}$ calmodulin; AB.BSA.nt: AB.BSA + $10 \mathrm{nM} 42$ nt random oligo sequences; AB.BSA.nt.Cam: AB.BSA.nt $+9 \mu \mathrm{M}$ calmodulin.

\section{Benzyl guanine (BG) conjugation of ss-DNA attachment strand}

Custom made 5' C6-amine modified oligonucleotides were covalently conjugated with benzylguanine NHS ester (BG GLA-NHS; NEB). Briefly, $170 \mu \mathrm{M}$ oligo was incubated with $3.2 \mathrm{mM}$ BG GLA-NHS in $100 \mathrm{mM}$ sodium borate $(\mathrm{pH} 8.5)$ at $37^{\circ} \mathrm{C}$ with rotation for 4 hours. The 
oligo was desalted on G-50 MicroColumns (GE Healthcare) and the oligo concentration was determined using UV abrsobance at $260 \mathrm{~nm}$ (Nanodrop). BG-oligo was stored at $4^{\circ} \mathrm{C}$ and used for protein labeling.

\section{Myosin VI expression, purification and conjugation with BG-oligo}

The procedures for Myosin VI expression and purification from Sf9 insect cells and the labeling of the motor with BG-ssDNA attachment oligo has been described by us previously. ${ }^{1,2}$ The Myosin VI (UniProt Accession number Q9UM54) construct consisted of an N-terminal motor domain and lever arm followed by a GCN4 leucine zipper motif to artificially dimerize the motor followed by a C-terminal SNAP and FLAG tags for oligo labeling and affinity purification respectively. An anti-FLAG resin based affinity purification was used to bind the protein from clarified Sf9 cell lysates. Myosin VI bound to anti-FLAG resin was incubated with excess $(>10 \mu \mathrm{M})$ of $\mathrm{BG}$ oligo overnight at $4^{\circ} \mathrm{C}$ with rotation for oligo labeling of the protein. Excess oligo was removed by washing the resin three times with buffer containing $20 \mathrm{mM}$ Imidazole $\mathrm{pH}$ 7.4, $3 \mathrm{mM}$ DTT, $1 \mu \mathrm{g} / \mathrm{ml}$ PMSF, $10 \mu \mathrm{g} / \mathrm{ml}$ aprotinin, and $10 \mu \mathrm{g} / \mathrm{ml}$ leupeptin. Myosin VI labeled with the BG-oligo attachment strand was eluted from the anti-FLAG resin with $0.2 \mathrm{mg} / \mathrm{ml}$ FLAG-peptide (Sigma) and was stored at $-20^{\circ} \mathrm{C}$ in $55 \%$ glycerol as a cryoprotectant.

\section{DNA Origami preparation}

The DNA strands used for origami preparation have been listed previously. ${ }^{1}$ Nanostructures were prepared by mixing single-stranded M13mp18 DNA (NEB) with excess of short staple strands (IDT) in TAE-Mg $\mathrm{Mg}_{2}+$ buffer $(40 \mathrm{mM}$ Tris, $20 \mathrm{mM}$ acetic acid, $1 \mathrm{mM}$ EDTA, 12.5 $\mathrm{mM} \mathrm{MgCl}_{2}$ ) followed by the annealing protocol listed in Supporting Information Table S1. Each scaffold contains $23 \mathrm{Cy} 5$ molecules for imaging and a biotinylated strand to facilitate conjugation of nanostructures to avidin-coated beads. 
Table S1: Annealing protocol for DNA nanostructures

\begin{tabular}{|c|c|c|}
\hline Temperature Range & Degree Change & Time Interval \\
\hline $90-80{ }^{\circ} \mathrm{C}$ & $0.1^{\circ} \mathrm{C}$ & $3 \mathrm{sec}$ \\
\hline $80-70{ }^{\circ} \mathrm{C}$ & $0.1{ }^{\circ} \mathrm{C}$ & $3 \mathrm{sec}$ \\
\hline $70-60{ }^{\circ} \mathrm{C}$ & $0.1{ }^{\circ} \mathrm{C}$ & $6 \mathrm{sec}$ \\
\hline $60-50{ }^{\circ} \mathrm{C}$ & $0.1{ }^{\circ} \mathrm{C}$ & $30 \mathrm{sec}$ \\
\hline $50-40{ }^{\circ} \mathrm{C}$ & $0.1{ }^{\circ} \mathrm{C}$ & $6 \mathrm{sec}$ \\
\hline $40-30{ }^{\circ} \mathrm{C}$ & $0.1{ }^{\circ} \mathrm{C}$ & $6 \mathrm{sec}$ \\
\hline $30-20{ }^{\circ} \mathrm{C}$ & $0.1{ }^{\circ} \mathrm{C}$ & $6 \mathrm{sec}$ \\
\hline $4{ }^{\circ} \mathrm{C}$ & Hold & Hold \\
\hline
\end{tabular}

\section{Purification of Myosin-Origami scaffolds for motility assay}

Cy5-nanostructures were incubated for 10-15 min with streptavidin-coated magnetic beads (NEB) at $30{ }^{\circ} \mathrm{C}$ with shaking in AB.BSA.nt buffer. Resin was washed three times with AB.BSA.nt and then incubated with excess (150 nM for saturably labeling 1-site origami) oligo conjugated Myosin VI for $10-15$ min at $30{ }^{\circ} \mathrm{C}$ with shaking. The origami labeled with Myosin VI was eluted off the streptavidin magnetic beads by incubation with $0.1 \mu \mathrm{M}$ of ssDNA elution strand in AB.BSA.nt.Cam buffer which binds to the biotinylated DNA strand on the origami and causes a strand displacement reaction to release the biotinylated strand from the origami.

\section{Motility assay with DNA origami on single actin filaments}

Motility assays were performed using plasma-cleaned glass coverslips coated with $0.1 \%$ colloidin in amyl acetate (Corning, 22 X $22 \mathrm{~mm}$ ). Flow chambers were prepared by adhering the colloidin coated glass coverslips to glass slides (Fisher Scientific, $3^{\prime \prime} \times 1^{\prime \prime} \times 1 \mathrm{~mm}$ ) using strips of double-sided tape. A biotin-neutravidin linkage was created on the surface by flowing in biotin-conjugated BSA at $1 \mathrm{mg} / \mathrm{ml}$ and neutravidin at $0.2 \mathrm{mg} / \mathrm{ml}$. Excess biotin and neutravidin was removed by three washes with AB.BSA. Biotinylated Alexa-488 F-actin filaments were adhered to the surface via the BSA biotin-neutravidin linkage. Purified myosin-DNA origami complexes in AB.BSA.CAM.nt supplemented with motility assay 
reagents $(2 \mathrm{mM}$ ATP, $1 \mathrm{mM}$ phosphocreatine, $0.1 \mathrm{mg} / \mathrm{ml}$ creatine-phospho-kinase, $25 \mu \mathrm{g} / \mathrm{ml}$ glucose-oxidase, $45 \mu \mathrm{g} / \mathrm{ml}$ catalase, $1 \%$ glucose, $1 \mu \mathrm{M}$ random library 42 -nt ssDNA) were added to the flow chamber. Motility of origami scaffolds was assayed at $1 \mathrm{~Hz}$ and $100 \times$ magnification on a Nikon Eclipse Ti inverted epifluorescence microscope.

\section{Coupling of Myosin Origami Scaffolds to Avidin Coated beads for Optical Trapping}

To prepare beads with DNA origami, $5 \mu \mathrm{l}$ of $1 \mu \mathrm{m}$ diameter (Spherotech, $1 \% \mathrm{w} / \mathrm{v}$ ) avidin coated beads are first spin washed twice $(16000 \mathrm{~g}, 1 \mathrm{~min})$ in $50 \mu \mathrm{l} \mathrm{AB}$ buffer and then once with $50 \mu \mathrm{l}$ of AB.BSA to passivate the bead surface. Then the beads are resuspended in $50 \mu \mathrm{l}$ of AB.BSA and incubated with $5 \mu \mathrm{l}$ of biotinylated DNA Origami stock for 10 minutes at $30^{\circ} \mathrm{C}$ in a dry incubator shaker. Excess unbound DNA origami are then removed with two washes of $50 \mu \mathrm{l}$ AB.BSA.nt. The bead-origami pellet is resuspended in $50 \mu \mathrm{l}$ of AB.BSA.nt and purified oligo-labeled Myosin VI is added at a concentration of $150 \mathrm{nM}$ and incubated for 10 minutes at $30^{\circ} \mathrm{C}$ in a dry incubator shaker. Excess unbound Myosin VI is removed with two washes of $50 \mu \mathrm{l}$ AB.BSA.nt.Cam and the origami-Myosin VI bound beads were finally resuspended in $50 \mu \mathrm{l}$ AB.BSA.nt.Cam. The final dilution of beads coupled to origami Myosin VI used in the optical trapping assay was made in AB.BSA.nt.Cam supplemented with motility assay reagents $(2 \mathrm{mM}$ ATP, $1 \mathrm{mM}$ phosphocreatine, $0.1 \mathrm{mg} / \mathrm{ml}$ creatine-phospho-kinase, $25 \mu \mathrm{g} / \mathrm{ml}$ glucose-oxidase, $45 \mu \mathrm{g} / \mathrm{ml}$ catalase, $1 \%$ glucose).

\section{Optical Trapping Assay}

The sample preparation procedure for optical trapping experiments was similar to that of motility assay where in the last step, avidin beads coupled with myosin-origami scaffolds was added in the mix instead of just the myosin-origami scaffolds keeping the other reagents and concentrations the same. 


\section{Optical Trapping Setup}

The experimental setup consists of a $1064 \mathrm{~nm}$ wavelength trapping laser source (Coherent Inc.) that passes through a two-axis acousto-optic-deflector (AOD, IntraAction Corp., DTD274HA6). The beam is expanded and steered into the microscope objective (Nikon 100x, 1.4 NA, oil immersion). Detection laser (Point Source Inc., iFLEX 2000, 50 mW, 830 nm, p-polarized) is added collinear to the trapping laser using a polarizing beam splitter (PBS) cube. Intensity of the detection beam is reduced by placing a neutral density filter (ND) in its path. Intensity is adjusted such that it is less than required to trap a bead. After passing through the sample, the beams are collected by a 1.25NA condenser (obtained from a bench microscope). The trapping laser is blocked using a laser line filter (Thorlabs, FL830-10) and the back-focal-plane image of the detection laser is imaged onto a quadrant photo-diode (Pacific Silicon Sensors, QP50-6SD2) with integrated amplifier circuit. The photodiode module provides three signals $\mathrm{Vx}, \mathrm{Vy}$, and $\mathrm{Vz}$ that represent asymmetry of light distribution on the photodiode along x coordinate, along y co-ordinate and the total intensity of light respectively (Fig.1). The signals are captured by a Field Programmable Gate Array (FPGA) based data acquisition card (National Instruments, 7833R). Control logic and voltage to position mapping is programmed on this hardware using custom written code in LabVIEW for FPGA.

\section{Custom MATLAB Software, Step Detection, and Data}

\section{Analysis}

Data obtained from optical trapping experiments was analyzed using a custom MATLAB software which takes in the timed bead position data from photodiode. Bead position data was first filtered by applying a $4^{\text {th }}$ order low pass Butterworth filter with cut-off frequency at $10 \mathrm{~Hz}$ in order to filter out the high frequency noise components. Filtered bead position data 
is then plotted against time. Events are identified by observing the shift in bead position outside of the trap via a series of stepping motion and then bead position suddenly falling back at the center of the trap indicating the detachment from actin filament. The variance of the bead position is also calculated in a moving window of 200 points and plotted on the same figure as bead position data. The events are also confirmed by observing the reduction in variance of bead position due to attachment of Myosin with Actin filament and increase in variance after detachment. Quantifying the reduction in variance upon filament binding of the motor gives a measure of linkage stiffness. For measuring the steps, the software prompts user to select the 4 points. The first two points determine the baseline position of the bead. The third and fourth point are chosen to be the starting and ending point of the bead at a new shifted baseline position. The difference between the mean position values of first two points and the mean position values of last two point gives the step size which is stored in an array. The difference between the time values of fourth point and third point gives the dwell time for the corresponding step which is also stored in an array along with corresponding value of step size. Steps with dwell time of less than 0.05 seconds are neglected in the analysis. Force values are obtained by multiplying the distance travelled out of the center of the trap with the corresponding stiffness values.

\section{References}

(1) Hariadi, R. F.; Cale, M.; Sivaramakrishnan, S. Myosin lever arm directs collective motion on cellular actin network. Proceedings of the National Academy of Sciences 2014, 111, 4091-4096.

(2) Hariadi, R. F.; Sommese, R.; Sivaramakrishnan, S. Tuning myosin-driven sorting on cellular actin networks. eLife 2015, 2015, 1-16. 